$R M x A C, \mathbf{5 3}, 8-13(2021)$

(C) 2021: Instituto de Astronomía, Universidad Nacional Autónoma de México

https://doi.org/10.22201/ia.14052059p.2021.53.03

\title{
THE 4 M NEW ROBOTIC TELESCOPE PROJECT: AN UPDATED REPORT
}

C. M. Gutiérrez ${ }^{1,2}$, M. Torres ${ }^{1,2}$, A. Oria ${ }^{1,2}$, J. J. Fernández-Valdivia ${ }^{1,2}$, D. Arnold ${ }^{3}$, D. Copley ${ }^{3}$, C. Copperwheat ${ }^{3}$, J. de Cos Juez ${ }^{4}$, A. Franco ${ }^{1,2}$, Y. Fan ${ }^{5}$, A. García Piñero ${ }^{1,2}$, E. Harvey ${ }^{3}$, H. Jermak ${ }^{3}$, X. Jiang ${ }^{6}$, J. H. Knapen ${ }^{1,2}$, A. McGrath ${ }^{3}$, A. Ranjbar ${ }^{3}$, R. Rebolo ${ }^{1,2,7}$, R. Smith ${ }^{3}$, I. A. Steele ${ }^{3}$, Z. Wang ${ }^{8}$, X. $\mathrm{Wu}^{9}$, D. $\mathrm{Xu}^{10}, \mathrm{~S} . \mathrm{Xue}^{10}, \mathrm{~W}$. Yuan ${ }^{10}$, and Y. Zheng ${ }^{11}$

\section{RESUMEN}

El nuevo telescopio robótico (NRT) es una colaboración internacional para construir y operar un telescopio completamente robótico de $4 \mathrm{~m}$ de diámetro. El telescopio aprovechará las excelentes condiciones atmosféricas en el Observatorio del Roque de los Muchachos (ORM), que junto a su gran apertura, su operación enteramente robótica, su rápida respuesta y un conjunto de versátil y variada instrumentación en el óptico e infrarrojo cercano garantiza una contribución científica de alto impacto enfocada principalmente en la novedosa área de la astronomía de dominio temporal. Este artículo presenta la motivación científica y el estado del proyecto, discutiendo posibles soluciones técnicas bajo evaluación para la óptica, la mecánica y el sistema de control.

\section{ABSTRACT}

The New Robotic Telescope (NRT) is an international collaboration to build and operate a 4m diameter fully robotic telescope. The telescope will take advantage of the superb atmospheric conditions at the Observatory of the Roque de los Muchachos (ORM). In conjunction with a large aperture, entirely robotic operation, quick response, and a set of versatile instrumentation in the optical and near-infrared this guarantees a high scientific impact focused mainly in the area of time domain astronomy. This paper presents the scientific motivation and the status of the project, discussing possible technical solutions under evaluation for the optics, mechanics and control system.

Key Words: telescopes

\section{INTRODUCTION}

The New Robotic Telescope (NRT) is an international collaboration that aims to build and op-

\footnotetext{
${ }^{1}$ Instituto de Astrofísica de Canarias, E-38205 Tenerife, Spain.

${ }^{2}$ University of La Laguna, E-38206 Tenerife, Spain.

${ }^{3}$ Astrophysics Research Institute, Liverpool John Moores University, IC2, Liverpool Science Park, 146 Brownlow Hill, Liverpool, L3 5RF, UK.

${ }^{4}$ Instituto Universitario de Ciencias y Tecnologías Espaciales de Asturias E-33004, C/Independencia 13-Oviedo, Spain.

${ }^{5}$ Yunnan Astronomical Observatories, Chinese Academy of Sciences, Kunming 650011, China.

${ }^{6}$ National Astronomical Observatories, Chinese Academy of Sciences, Beijing 100101, China.

${ }^{7}$ Consejo Superior de Investigaciones Científicas, E-28006 Madrid, Spain.

${ }^{8}$ Shanghai Astronomical Observatory, Chinese Academy of Sciences, Shanghai 200030, China.

${ }^{9}$ Purple Mountain Observatory, Chinese Academy of Sciences, Nanjing 210008, China.

${ }^{10}$ CAS Key Laboratory of Space Astronomy and Technology, National Astronomical Observatories, Chinese Academy of Sciences, Beijing 100101, China.

${ }^{11}$ National Astronomical Observatories, Nanjing Institute of Astronomical Optics and Technology (NIAOT), Chinese Academy of Science, 188 Bancang Street, Nanjing 210042, China.
}

erate an entirely robotic telescope with a diameter of $4 \mathrm{~m}$. The project started about five years ago with Liverpool John Moores University (LJMU, UK) and the Instituto de Astrofísica de Canarias (IAC, Spain) as promoters, and the Instituto Universitario de Ciencias y Tecnologías Espaciales de Asturias (ICTEA, Spain), the National Astronomical Observatories, Chinese Academy of Sciences, China (NAOC) and the National Astronomical Research Institute of Thailand (NARIT) incorporated recently to the project. Currently, the project is finishing its conceptual design to enter a more advanced design phase with the aim to start operation in about five years time.

The motivation of the project is to develop an astronomical facility that will play a relevant role in the forthcoming era of time domain astronomy by providing key complementary observations for follow-up programmes in order to identify and characterize the most promising targets discovered by other large facilities. Previous experience within the consortium building and operating the $2 \mathrm{~m}$ Liverpool robotic telescope (Steele et al. 2004), and developing forefront astronomical instrumentation is a solid base for a proper execution of the project. The strength of the 
NRT will rely on having the largest collector area for a telescope working in robotic mode, a set of at least five instruments simultaneously installed in the different focal stations, an efficient system to evaluate the priority of the targets and a quick response that is essential for some of the scientific cases.

The telescope will be sited at the Carlsberg Meridian site on the Observatorio del Roque de Los Muchachos (ORM) in La Palma (Canary Islands, Spain) at 2,400 $\mathrm{m}$ altitude. Extensive site testing campaigns for over thirty years have demonstrated (Wilson et al. 1999), that the ORM has excellent conditions for astronomical observations, e. g. a mean seeing of $0.69 \operatorname{arcsec}(\sim 20 \%$ of the time below 0.5 arcsec $)$ and an extremely dark sky $(\sim 22 \mathrm{mag}$ $\operatorname{arcsec}^{-2}$ in V), at the level of the best places in the world. The location allows access to $\sim 3 / 4$ of the sky, sharing $\sim 10,000$ square degrees with Southern hemisphere facilities.

This contribution presents a brief updated report on some of the most relevant analysis conducted within the current conceptual design phase of the project. Previous reports of the project have been presented by Copperwheat et al. (2015) and Gutiérrez et al. (2019). We refer readers to those reports for further details of the project and in particular for topics like scientific cases, site, dome concepts and instrumentation scarcely considered here.

The paper is structured as follows: after this introduction, $\S 2$ enumerates some of the most interesting scientific cases; $\S 3$ and $\S 4$ are devoted to the optics and optomechanics of the telescope, whilst $\S 5$, $\S 6$ and $\S 7$ sketch the overall concept of the mechanical structure, the control system of the telescope and the enclosure respectively. $\S 8$ presents the conclusions.

\section{SCIENTIFIC MOTIVATION}

The NRT will be very well suited in terms of collecting area ( $4 \pi$ square meters) and quick response $(\sim 30 \mathrm{sec}$ on target) taking an intermediate position between the large ( $>8 \mathrm{~m}$ telescopes) and other existing or planned fully robotic telescopes $(\sim 1-2 \mathrm{~m})$. Although the NRT has been conceived as a multipurpose telescope with instrumentation covering most of the observing modes in the full optical and nearinfrared ranges, the scientific motivation and therefore most of the observing time will likely be dedicated to the area of time domain and multimessenger astronomy. For instance, programmes to validate models of supernovae detected by, e. g., the LSST http://lsst.org) or to measure their light curves could be efficiently conducted by NRT. A quick re- sponse will also be essential to observe the optical afterglows of gamma ray bursts (GRBs). Space missions like the Einstein Probe (Yuan et al. 2016) of the Chinese Academy of Sciences will discover various types of high-energy transients in the soft X-ray band in large numbers, including tidal disruption events (TDEs), supernova shock breakouts, highredshift GRBs providing a huge number of targets for NRT identification and characterization. The size and robotic operation of the NRT is also ideal to study exoplanets by long follow-up programmes with appropriate instrumentation to measure, for instance, the variations in the polarization of reflected light of exoplanets along the orbital phase.

The fully autonomous way of NRT operation will allow also to conduct a plethora of scientific cases that require multiwavelength follow-up observations in coordination with other facilities. Likely objects selected from the Gaia catalogs (Gaia Col. 2016) will be one of the main sources of targets.

NRT observations will also be very relevant to identify and characterize the electromagnetic counterparts of gravitational waves (Abbott et al. 2016) and astronomical neutrinos (ICE Col. 2018).

\section{OPTICAL DESIGN}

Some of the main NRT scientific cases related with transient objects (see above) require a quick response. This has been taken into account in the optical and mechanical design, favoring the selection of a compact structure that facilitates the telescope dynamics. However, that concept increases the sensitivity of the system to mirror alignment, so a balance had to be achieved between the optical and mechanical configurations.

Initially, a f/7.5 Ritchey-Chrétien (Wetherell \& Rimmer 1972) configuration was considered, with a fast f/1.75 primary mirror M1, i. e., a compact tube with a length of $\sim 7 \mathrm{~m}$. A representation of this layout can be seen in Figure 1. Although this configuration provides a very compact structure, it turned out to be very sensitive to alignment, and the required mirror quality pushed the limits of the state of the art. That motivated us to consider alternative designs like the Dall-Kirkham (Beach \& Rakich 2005) with a spherical secondary mirror M2. This concept provides a system which is an order of magnitude less sensitive to mechanical deformations and thermal effects, and is comparatively much easier to collimate and align. A spherical secondary mirror also reduces the complexity of manufacturing as compared to the hyperbolic mirror in the RitcheyChrétien design. The relatively worse performance 


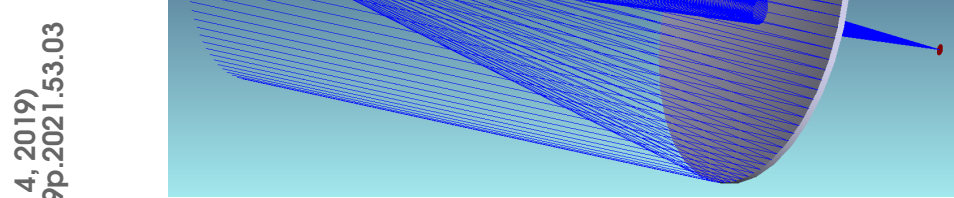

Fig. 1. NRT optical design for a f/7.5 Ritchey-Chrétien configuration.

off-axis and the need for a more powerful corrector in the Dall-Kirkham optics as compared to the RitcheyChrétien, may be assumed for a telescope like NRT that will be dedicated mainly to spectroscopy. An ongoing in deep analysis of performance, sensitivity, manufacturing complexity and cost of both optical systems will help to reach a final decision.

\subsection{Segmented Primary Mirror}

Monolithic primary mirrors are the common choice for 4m-class telescopes, e. g. the WHT (Benn 2014), the SOAR (Krabbendam et al. 2004), and the DCT (Levine et al. 2018). However, segmented mirror topologies initially developed to overcome the maximum practical size of monolithic mirrors (about $8 \mathrm{~m}$ ), are starting to be applied to smaller apertures, e. g. the LAMOST (Cui et al. 2010) and the SEIMEI (Jikuya et al. 2019) telescopes, due to a number of advantages in terms of manufacturing, cost, weight and recoating. In fact, a segmented approach allows to reduce the weight of the primary mirror which is an important contributor to the total telescope mass. It also reduces the telescope downtime associated to mirror coating, and facilitates all the mirror handling operations. Furthermore, there is a broader choice of mirror manufacturing options for smaller mirrors, and a segmented design is scalable to larger apertures.

Several options for the segmented topology of the primary mirror of NRT are under study and evaluation. Some of them, based on arrangements of hexagonal and circular mirrors, are shown in Figure 2. The performance of the telescope and the sensitivity to segment alignment are being evaluated for each topology. In general, hexagonal segments allow for more compact apertures, but present more difficulties to manufacture. Circular segments are
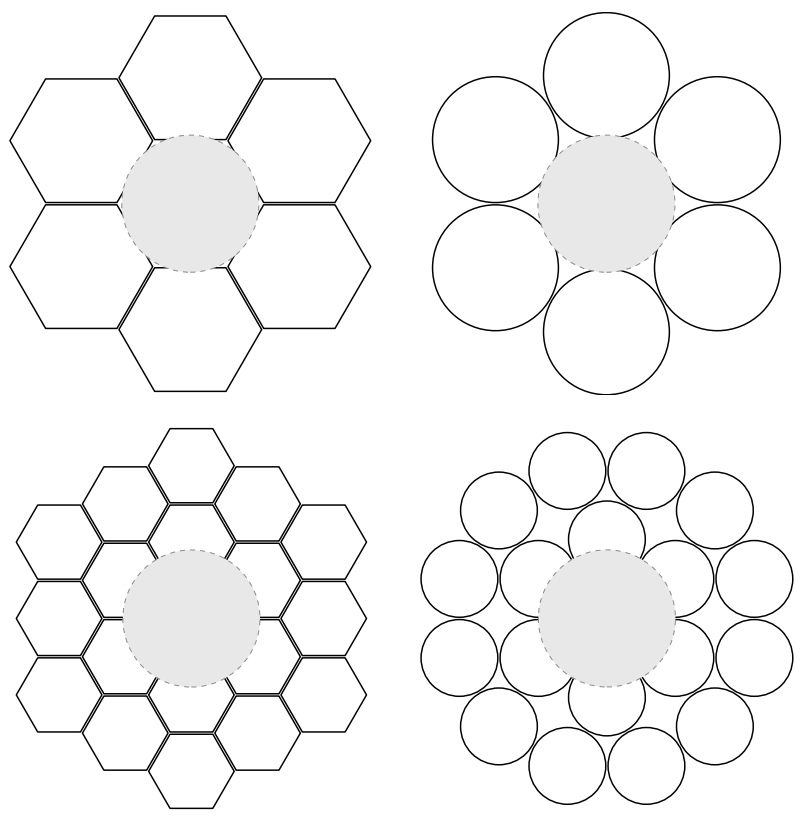

Fig. 2. NRT primary mirror segmented topologies, for 6 and 18 hexagonal and circular segments. The shaded region in the center of each configuration corresponds to the area obscured by the secondary mirror.

easier to manufacture but the optical performance of the telescope is worse in the near infrared. Other considerations affecting each topology are considered in next sections.

\section{OPTOMECHANICS}

This section presents the different alternatives that are under consideration for the optomechanics of the primary and secondary mirror assemblies of the telescope.

\subsection{Mirror Support System}

The deformation of the reflecting surfaces of the mirrors has to be controlled to meet the telescope optical error budget. That requires an adequate selection of the substrate material (Zerodur in our case), the mirror geometry and the support system in order to avoid transmitting the deformations produced from the mirror interfaces to the optical surface.

Pointing to different altitudes requires a mirror support system able to sustain the mirror weight in both the axial and lateral directions at any altitude. The challenges on how to support gravity are very different in those two directions, and for this reason the support system of the mirrors is separated into two almost independent components, each of them stiff in one direction and compliant in the other. The 


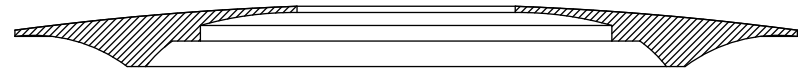

Fig. 3. Section view of the M2 substrate, showing the double-arch lightweighting solution.

baseline design for both the M1 segments and the M2 mirror is the general solution adopted in other segmented telescopes with a mechanical whiffletree for axial support and a central diaphragm for lateral support.

In parallel to this analysis, a preliminary study of different lightweighting options for the M2 mirror has been performed. The objective of a lightweighted M2 is to reduce the overall tube weight and to match the hexapod specifications to an available commercial solution. In the scope of this first study, simulations to optimize the geometry of the M2 substrate minimizing the axial gravity print-through and the mirror mass have been conducted. The results of the simulations have led to the concept presented in Figure 3. This M2 design is based on a double arch substrate with a reduced weight of about $100 \mathrm{~kg}$, which represents a lightweighting factor of $\sim 50 \%$.

\subsection{Active Optics}

Active optics is essential to relax the requirements of the telescope structural deformations, therefore allowing for a lighter structure. Such active corrections will be incorporated into NRT by M1 and M2 mirror repositioning. In the case of a segmented configuration, The M1 active optics system will be comprised by three linear actuators per segment operating in a closed loop with a set of sensors that guarantee the relative position between mirrors and keep the global shape of the M1 optical surface. The alignment between the M1 assembly and the M2 mirror will be actively controlled with the M2 hexapod.

Topologies with one ring of six circular or hexagonal segments (see Figure 2) have less system complexity and easier active optics control as compared to the 18 segments ones, but obviously they require bigger and heavier segments. On the other hand, topologies with a higher number of segmented mirrors have more correction capabilities to the overall primary mirror shape and they also have broader options for COTS (commercial of the shelf) segment actuators due to the smaller size and weight. If COTS solutions are finally adopted, the efforts will be directed to a proper definition of the specifications of the systems during the telescope preliminary design.

\section{TELESCOPE STRUCTURE}

The elevation structure will be an open truss with two different variants under consideration: a classical Serrurier and a tripod-like structure. As discussed in the optomechanics section, active optics will be implemented for the telescope so the ability to maintain collimation by passive means is not mandatory and then the tube deformation requirements can be relaxed. The classical Serrurier has the benefit of reducing the M1 obscuration, however the tripod is structurally advantageous, lighter and the obscuration problem can be limited if the tube beam locations are matched with the spaces naturally present in a segmented telescope. A light upper tube (with a lightweighted M2 and a tripod-like tube) moves the M1 assembly closer to the elevation axis to balance the tube center of gravity and permits a direct attachment to the elevation ring. Three proposals are being considered for the azimuth mount: conventional yoke (baseline), gantry and rocking chair.

The telescope structure design has to include the slewing time assessment as quick response is one of the key features of the telescope. A preliminary model has confirmed that the slew requirements can be fulfilled with commercially available solutions. That model considers both the slew kinematics and the associated settling time assuming a very simple single degree of freedom model subjected to viscous damping. Two motion profiles have been evaluated: a constant acceleration profile (trapezoidal in velocity) and an S-curve profile of minimum jerk that limits the settling times. Figure 4 shows a comparison of the time required to slew and settle the tube position for both profiles and different peak accelerations. The dynamic inverse problem has also been solved for each axis to compute the required driving torques, predict slew time for a given motor, and in this way establish an admissible maximum length for the telescope tube. A complete trade-off of the different configurations is still to be performed.

\section{CONTROL SYSTEM}

The telescope control system (TCS) is a key concept in the design of NRT. The TCS will be responsible for controlling, coordinating, monitoring and planning all systems (hardware and software) involved in the operation of the telescope, it must guarantee the proper operation of all systems and services and the security in terms of fault tolerance, problem recovery and safe shutdown in the event of a serious failure. A fully autonomous operation requires a constant exchange of information between the different subsystems and the TCS in order to 


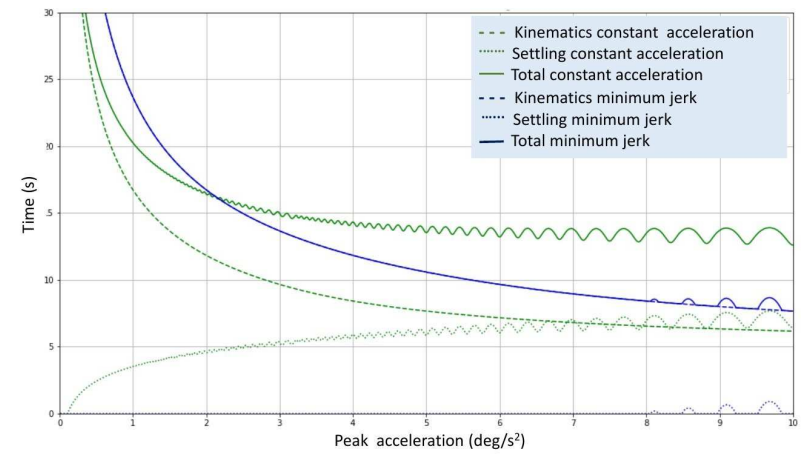

Fig. 4. Blind pointing time vs peak acceleration for a 70 degrees slew, comparing the trapezoidal (green) and the S-curve (blue) profiles.

determine and execute at any moment the most adequated actions. This requires quick responses and to establish a set of priorities according to the state of the telescope and its environment, e. g., atmospheric conditions at any moment.

The NRT control system architecture aims to follow best practices in services decoupling and deployment, following recent techniques in containerization and orchestration. This type of system will give a great stability, scalability, and flexibility, allowing new services to be added or removed without affecting the operation of the others.

The current robotic telescopes in operation have simple TCSs as compared to the requirements of the NRT. Therefore, in this phase of conceptual design we are evaluating pros, cons, suitability and efforts needed to adopt a new solution. There are several platforms, such as ROS (Robotic Operating System, https://www.ros.org/), INDI (Instrument Neutral Distributed Interface, https://www.indilib.org/), ACS (http: //www . eso.org/ almamgr/AlmaAcs/index.html) or TANGO (https://www.tango-controls.org/), which offer a base to develop a TCS. However, independently of their relative value and suitability for NRT, all of them require a high effort in implementation, adaptation and verification. As a promising alternative to those systems, we are exploring the possibility to adapt GCS, the control system of GTC (Filgueira \& Rodríguez 1998), running successfully in that telescope and having a development team with many years of experience. The paragraphs below describe the main facts of GCS and the inherent problems we face to adapt it to NRT.

GCS has advantages like the execution of processes in kernels with real time, a simulator and a development platform, as well as a good log record to detect and correct problems. However, it also has some disadvantages with respect to other platforms: it uses CORBA (Common Object Request Broker Architecture, https: //www. corba. org/system) which is a bit outdated and perhaps GCS is too complex for the NRT requirements. An implementation using DDS (Data Distribution Service, https://www.dds-foundation.org/) might be more convenient.

GCS is a complex system, and has thus been decomposed into different sets of subsystems that are formed by groups of devices. These subsystems are responsible for the operation of the telescope performing the tasks assigned to them: Logging \& Alarms Service, Observing Engine, System Monitor, Data Factory, Sequencer, Scheduler, Acquisition \& Guiding Control System (AGCS), Science Instrument Control System (SICS), Enclosure and Services Control System (ESCS), Configuration Service, Primary Mirror Control System (M1CS), Interlock \& Safety Control System (ISS), Main Axis Control System (MACS), etc.

The communications between objects are via a network using an implementation of the CORBA standard for these devices. Within this model, a client device can invoke a method exported by another server device with location transparency. This structure allows for a great robustness, flexibility and fault tolerance. The devices are defined using a plain text file, from which the development tools generate a skeleton in $\mathrm{C}++$ or Java. The generation of this code skeleton is done with a command line tool. The user-written source code may be regenerated multiple times without affecting previous compilations. As in object-oriented programming, devices can inherit from other devices, and there is also the possibility of grouping several devices to create a more complex structure. The devices inherit the CORBA communication interfaces and methods of the base class, which allows them to communicate with the other services and subsystems of the control system. This feature allows the devices to interact with the rest of the system to connect to the databases, obtain information on the status of the telescope, be aware of alarms, and allow the control system to know the status of the device at any time.

GCS currently does not implement any subsystem for robotic control, since the GTC works in the traditional way with human operation. The challenge of adapting GCS to the NRT control system will be the integration of the robotic control of the telescope, allowing a completely autonomous operation. 